\title{
Decision time for clinical decision support systems
}

\author{
Authors: Dympna O'Sullivan, ${ }^{A}$ Paolo Fraccaro, ${ }^{B}$ Ewart Carson ${ }^{C}$ and Peter Weller ${ }^{D}$
}

Clinical decision support systems are interactive software systems designed to help clinicians with decision-making tasks, such as determining a diagnosis or recommending a treatment for a patient. Clinical decision support systems are a widely researched topic in the computer science community, but their inner workings are less well understood by, and known to, clinicians. This article provides a brief explanation of clinical decision support systems and some examples of real-world systems. It also describes some of the challenges to implementing these systems in clinical environments and posits some reasons for the limited adoption of decision-support systems in practice. It aims to engage clinicians in the development of decision support systems that can meaningfully help with their decisionmaking tasks and to open a discussion about the future of automated clinical decision support as a part of healthcare delivery.

KEYWORDS: Clinical decision support systems, decision-making, diagnosis

\section{Context}

Information technology (IT) is now commonplace in almost every branch of healthcare. Electronic health records, eprescribing and digital medical imaging are well known to clinicians and have been implemented with varying degrees of success. ${ }^{1}$ In addition, clinicians increasingly make use of online repositories such as PubMed and Google Scholar, ${ }^{2}$ and specialised search engines such as FindZebra ${ }^{3}$ to help answer clinical questions. One often overlooked set of IT tools are clinical decision support systems (CDSSs), which have been defined as systems that 'provide clinicians or patients with computer-generated clinical knowledge and patient-related information, intelligently filtered or presented at appropriate times, to enhance patient care'. ${ }^{4}$ Such CDSSs have been the subject of academic computer science research for more than

Authors: Asenior lecturer in health informatics, Centre for Health Informatics, City University London, UK; Bresearch fellow, Centre for Health Informatics, City University London, UK; Cemeritus professor of systems science, Centre for Health Informatics, City University London, UK; D senior lecturer in health informatics, Centre for Health Informatics, City University London, UK
50 years ${ }^{5}$ and offer the potential for better supported decision making by clinicians, improved compliance with medical standards and improved clinical efficiency and safety. ${ }^{6,7}$ Nonetheless, utilisation of CDSSs remains limited, and most healthcare IT systems do not include robust CDSS functions that can be widely employed across organisations, clinical presentations and domains. ${ }^{8}$

Some of the challenges to implementation of CDSSs relate to the volume of high-quality data required for state-of-the-art systems, the translation of such data to machine-readable states and the mapping of CDSS processes to fit with existing clinical workflows. As a result, successful implementation of CDSSs has tended to be site and domain specific, with major difficulties replicating these successes more extensively throughout healthcare systems. ${ }^{9}$ This is in contrast to commercial fields such as finance, where decision support technologies have been widely deployed. For example, risk-profiling tools for financial experts have been developed as easy-to-use programs that can assimilate information and guide users through complex financial information and associated decisions tailored to individual customer needs. Healthcare decision making is significantly more complex than financial planning; however, some of the challenges in both domains are similar: large quantities of data need to be linked, integrated and translated to machine readable formats, and expert knowledge is required to contextualise and apply the data in a meaningful way. We discuss some reasons for the limited dissemination and adoption of CDSSs to date and reflect on the major barriers that need to be overcome for these useful tools to be adopted more widely.

\section{Brief taxonomy of clinical decision support systems}

Clinical decision support systems vary widely in their type and complexity. Systems can be passive (users explicitly make a request for support), semi-active (watchdog systems are invoked automatically and present information when users request it) or active (triggered automatically, present information without it being requested and, in some cases, make decisions without the intervention of clinicians). They have been implemented to support clinicians across the spectrum of medical specialties and have been customised for different levels of clinical expertise from novice (eg student nurses) ${ }^{10}$ through nonspecialist (eg in community hospital settings) ${ }^{11}$ to highly specialist healthcare professionals (eg digital pathology). ${ }^{12}$ Simple CDSSs usually check the input provided by a clinician and verify whether the value is allowable or within a specified 
range and whether there are any predefined contraindications. The output of the CDSS is usually an alert or reminder. Examples of such CDSSs are usually embedded in order entry systems and include functions to provide basic guidance on dosing and to check drug allergies, duplicate therapies and drug-drug interactions. ${ }^{13}$ Mid-level CDSSs include prognostic calculators and automated clinical practice guideline systems. Prognostic calculators are used to automatically determine prognosis, usually by implementing established clinical scoring systems. Examples include the glioblastoma multiforme (GBM) calculator, which implements the European Organisation for Research and Treatment of Cancer (EORTC)'s scoring system ${ }^{14}$ and Adjuvant! Online, which is a tool to help decisions about adjuvant therapy in patients with early invasive breast cancer. ${ }^{15}$ Automated clinical practice guideline systems represent clinical knowledge from practice guidelines in one of a number of guideline modelling languages, which allows a CDSS to execute extracted guideline rules or algorithms to compute decisions about possible diagnoses or interventions. By coupling a computer-based guideline system with an electronic health record, recommendations can be personalised to the individual patient. Guideline-based CDSSs have been developed for a range of clinical specialties ${ }^{16}$ and are also widely used as a basis for helplines, where less expensive or less experienced clinical staff perform triage services, and telemedicine services, where diagnoses are performed remotely. ${ }^{17}$ These systems are also gaining traction in the consumer health informatics arena as the basis for online self-assessment tools for patients (eg NHS Choices Symptom Checker). ${ }^{18}$

Complex CDSSs use artificial intelligence, data mining or statistical methods to reason about the classification or prediction of a disease or patient state. These methods automatically identify key features that are important for the clinical classification or prediction problem and use mathematics to determine the way in which these features should be combined to create an output that represents the classification or prediction. ${ }^{19}$ Commonly used techniques include logistic regression, artificial neural networks and support vector machines. These complex methods have been applied to a wide range of clinical decision-making problems, including diagnosis of prostate cancer, ${ }^{20}$ screening for obstructive sleep apnoea in people with ischaemic heart disease ${ }^{21}$ and identification of psychiatric problems. ${ }^{22}$

In systems of simple or mid-level complexity, the decision computed by the CDSS can usually be easily explained to the clinician (eg by showing a trace of the rules used to compute the outcomes). In contrast, decisions in complex systems are computed using advanced mathematics and non-linear transformations, so it is difficult to document how specific decisions are reached and thus to explain the output to clinicians. However, these complex CDSSs better mirror clinicians' decision-making processes by integrating and reasoning over multiple facets of data from a patient and computing a likely outcome for a specific patient state.

\section{Challenges to implementation and adoption of clinical decision support systems}

Most literature focuses on operational aspects that act as a barrier to implementation of CDSSs. ${ }^{23}$ In particular, slowly emerging standards for healthcare IT and poor interoperability between clinical systems limit the development of generic, reusable and scalable CDSSs. However, valuable work is being carried out to remedy these technical gaps by standards agencies such as Health Level Seven International (HL7), ${ }^{24}$ and work on developing comprehensive biomedical terminologies such as Systematized Nomenclature of Medicine-Clinical Terms (SNOMED CT) is ongoing. ${ }^{25}$ Furthermore, the increasing prevalence of electronic health records should improve accuracy of and standardisation in data collection, as well as link disparate parts of patient records. These developments have important implications for CDSSs, which require accurate and comprehensive data to operate optimally, and standardised data to operate in different settings. We posit that these developments will ease practical development of CDSSs; however, other significant issues not related to technology will still present challenges. These challenges are related to so-called 'softer' elements and include vendors and users of systems, as well as organisational, legal and ethical challenges.

A survey of the CDSS capabilities of major commercial clinical information systems (CISs) in the USA found that most have small-scale, in-built functionalities - mostly comprised of alerts and reminders - with scant support for more complex decision-making tasks. ${ }^{26}$ The reason for the emphasis on simpler functionality is clear: in order for commercial systems to be viable, they need to scale to different contexts and work environments, so providers focus on simpler tasks that are homogeneous across institutions (eg computerised order entry). The development of CDSSs to support more complex decision making (eg prediction of patient states or classification of diseases) is significantly more difficult and time consuming, and such efforts have largely remained confined to academic environments, where researchers have the advanced computational expertise and time required to create appropriate solutions. However, the nature of these academic projects is such that they are funded for relatively short periods of time and, if deployed clinically, they usually remain standalone small-scale systems used only by the clinicians who were involved in their development. This is exacerbated by the fact that complex CDSSs are difficult to customise to different tasks and contexts, as they use specific learning algorithms that have been trained to achieve optimal accuracy on a specified set of patient attributes and states (eg different CDSSs are often required for paediatric and adult conditions, and systems may not generalise for similar patient cohorts from different countries). ${ }^{15}$ Wider deployment of any CDSS will also include the requirement to tailor the system to the local clinical setting, including the established clinical workflow, the site-specific clinical vocabulary and locally installed hardware and software IT systems. In addition, maintenance presents a significant challenge both in the face of rapidly advancing clinical knowledge and the lack of standardised institutional guidelines on periodic review of CDSSs. The maintenance dilemma is also intensified by the fact that many graduates who develop academic CDSSs tend to find limited opportunities in the healthcare domain and find more lucrative opportunities developing decision support tools for finance or industry. A common criticism of CDSSs is their poor usability. ${ }^{27}$ Clinicians work in busy environments with demanding 
time (and other) pressures, and any system that adds to those burdens will not be accepted. Reviews of clinicians' information-seeking behaviour show that a lack of time and formal training with IT systems - as well as having to distract from the current workflow and clinical task at hand because decision-making software is often not embedded directly within relevant CISs - resulted in clinicians using colleagues as their first source when seeking information to guide decisions. ${ }^{28}$

As already mentioned, the most commonly available CDSSs are alert and reminder systems, and even such rudimentary systems are frequently ill designed - for example, providing alerts that appear too frequently or that are not sufficiently specific and thus impede the clinical workflow. More complex CDSSs - for example, those that employ so-called 'black-box' methods such as neural networks from artificial intelligence - come with a different set of usability issues; these include a lack of understanding of these methods on the part of clinicians and an associated lack of comprehensibility about how the CDSS has computed its decision. However, these methods are necessary for the successful development of robust CDSSs that can reason over large clinical datasets, as well as to incorporate clinical knowledge in order to compute accurate outputs. These methods will gain in importance as complex, high-volume, genomic data become commonplace in clinical practice, and application of these techniques will play an important role in achieving the benefits of personalised medicine. ${ }^{29}$

An important tenet of human computer interaction - the domain of computer science that deals with the interaction between users and computers - is to design with the end user in mind. In the case of CDSSs, computer scientists often develop software to manipulate complex clinical concepts that they do not fully understand for clinicians who clearly understand these concepts but who may not well understand the technologies and methods that underpin CDSSs. It is clear that the most effective CDSSs will be developed through close collaboration between computer scientists and clinicians, which will allow computer scientists to gain better understanding of the real clinical needs that CDSSs should satisfy and will help clinicians to better understand the 'inner workings' of CDSSs. Sophisticated CDSSs - beyond alerts and reminders - require better informed end users, and, with the growing prevalence of computers in medicine, clinicians must be better supported so that their informatics training needs are met, thereby fostering understanding and trust in computerised knowledge used to generate decisions. Better motivation on the part of clinicians to use CDSSs would also drive commercial development, and vendors of CISs would be forced to expend more investment in CDSS technologies.

Although closer collaboration between the medical and computer science communities is required for useful and usable CDSSs, it must be recognised that these communities have different foundations. Medicine is a long established, highly regulated discipline, whereas no licence is required to practice computer science, but computer system errors could have a direct impact on patient safety. Although the US Food and Drug Administration has developed guidance regarding medical software and other safety-critical healthcare IT systems, software validation is not usually subject to protocols as stringent as other healthcare interventions (eg randomised controlled trials for new therapies). Furthermore, computer scientists usually work in teams who collectively develop software, whereas an individual clinician is often solely responsible for medical decisions related to their patients' health. Questions therefore arise about the legal risks to clinicians when relying on decisions generated by a CDSS, particularly when these systems use complex 'black-box' methods. On the other hand, CDSSs also have the potential to decrease medical litigation, because CDSSs that embed best practices, clinical guidelines and recommended standards of care could be used as a focus for quality control, including auditing of clinical decisions.

It is worth noting that, until recently, the vast majority of CDSS developments have been aimed at clinicians; however, increasing patient engagement with online medical content is giving rise to a new market for consumer-oriented CDSSs. A recent survey of 1,132 patients in the USA showed that $67 \%$ were favourably disposed to using online health resources as a complement to in-person doctor visits, and $47 \%$ would use them as a substitute..$^{30}$ On the one hand, consumer-oriented CDSSs have the potential to encourage patients to become more involved in and informed about their care, and can encourage them to make healthy choices to improve or maintain their health. It may also be possible to use these systems to relieve pressures on frontline services by allowing patients to self-manage some conditions. On the other hand, there are justifiable concerns about patients' ability to interpret medical information correctly and the quality of online health information. A recent study that assessed the quality of 60 mobile phone 'apps' providing health promotion in the areas of 'healthy diet', 'obesity', 'smoking cessation' and 'cancer prevention' found that less than 5\% of the apps indicated that the educational material was peer reviewed by appropriate clinical parties. ${ }^{31}$ Clinical decision support systems for consumer health informatics clearly raise many questions for the medical and computer science communities, and this is an area in which collaboration between both parties is essential to ensure the safe delivery of services within this new healthcare paradigm.

\section{Questions for clinical decision support systems' stakeholders}

From a technical standpoint, the time is now ripe to develop CDSSs that offer meaningful support for clinical decision making. The prevalence of healthcare information technologies that lend themselves to the development of CDSSs that can support complex decision making as part of clinical workflow including electronic health records, standards such as HL7 for sharing and integrating patient data, and mobile applications to aid with point-of-care consultations - is growing. However, other important challenges remain, and we conclude with some food for thought on the future of CDSSs:

> The computerisation of healthcare is something that is only going to increase and hence the clinician's educational needs must include generally relevant areas of IT. Should clinicians also be educated in the specific computer science methods that underpin CDSSs?

> If clinicians gain more knowledge of, and trust in, CDSSs, are vendors of CISs ready to change their approach and focus on the decision-support needs of clinicians in addition to operational tasks? 
> The decisions computed by complex CDSSs using techniques from artificial intelligence are often not transparent and there is a lack of institutional guidance on decision making supported by CDSSs. Should developers of CDSSs bear some responsibility for decisions taken by clinicians based on suggestions from CDSSs?

> Finally, if all challenges outlined in this viewpoint were addressed and it became possible to develop CDSSs such that their performance was on a par with the expert clinician, would clinicians want to use them?

\section{References}

1 Kaplan B, Harris-Salamone KD. Health IT success and failure: recommendations from literature and an AMIA workshop. J Am Med Inform Assoc 2009;16:291-9.

2 Shariff SZ, Bejaimal SA, Sontrop JM et al. Retrieving clinical evidence: a comparison of PubMed and Google Scholar for quick clinical searches. J Med Internet Res 2013;15:e164.

3 Dragusin R, Petcu P, Lioma C et al. FindZebra: a search engine for rare diseases. Int J Med Inform 2013;82:528-38.

4 Osheroff JA, Teich J, Levick D et al. Improving Outcomes with Clinical Decision Support: An Implementer's Guide, 2nd edn. Chicago: Healthcare Information and Management Systems Society (HIMSS), 2005.

5 Kulikowski CA, Weiss SM. Representation of expert knowledge for consultation: the CASNET and EXPERT projects. Boulder: Westview Press, 1982.

6 Jaspers MW, Smeulers M, Vermeulen H, Peute LW. Effects of clinical decision-support systems on practitioner performance and patient outcomes: a synthesis of high-quality systematic review findings. J Am Med Inform Assoc 2011;18:327-34.

7 Kushniruk AW, Bates DW, Bainbridge $M$ et al. National efforts to improve health information system safety in Canada, the United States of America and England. Int J Med Inform 2013;82:149-60.

8 Sittig DF, Wright A, Osheroff JA et al. Grand challenges in clinical decision support. J Biomed inform 2008;41:387-92.

9 Lyman JA, Cohn WF, Bloomrosen M, Detmer DE. Clinical decision support: progress and opportunities. J Am Med Inform Assoc 2010;17:487-92.

10 Hoffman K, Dempsey J, Levett-Jones T et al. The design and implementation of an interactive computerised decision support framework (ICDSF) as a strategy to improve nursing students' clinical reasoning skills. Nurs Educ Today 2011;31:587-94.

11 Farion K, Wilk S, Michalowski W et al. Comparing predictions made by a prediction model, clinical score, and physicians: pediatric asthma exacerbations in the emergency department. Appl Clin Inform 2013;4:376-91.

12 Shina H, Markey MK. A machine learning perspective on the development of clinical decision support systems utilizing mass spectra of blood samples. J Biomed Inform 2006;39:227-48.

13 Saverno KR, Hines LE, Warholak TL et al. Ability of pharmacy clinical decision-support software to alert users about clinically important drug-drug interactions. J Am Med Inform Assoc 2011;18:32-7.

14 Michaelsen SR, Christensen J, Grunnet K et al. Clinical variables serve as prognostic factors in a model for survival from glioblastoma multiforme: an observational study of a cohort of consecutive nonselected patients from a single institution. BMC Cancer 2013;13:402.
15 Adjuvant! Online, 2011. www.adjuvantonline.com/index.jsp [Accessed 28 February 2014].

16 Peleg M. Computer-interpretable clinical guidelines: a methodological review. J Biomed Inform 2013;46:744-63.

17 Stacey D, Macartney G, Carley M, Harrison MB. Development and evaluation of evidence-informed clinical nursing protocols for remote assessment, triage and support of cancer treatment-induced symptoms. Nurs Res Pract 2013;2013:171872.

18 NHS Choices. Symptom Checker, 2014. www.nhs.uk/symptomcheckers/ pages/symptoms.aspx [Accessed 24 June 2014].

19 Greenes RA. Clinical decision support: the road ahead. Amsterdam, Boston: Elsevier Academic Press, 2007.

20 Lee HJ, Hwang SI, Han SM et al. Image-based clinical decision support for transrectal ultrasound in the diagnosis of prostate cancer: comparison of multiple logistic regression, artificial neural network, and support vector machine. Eur Radiol 2010;20:1476-84.

21 Laporta R, Anandam A, El-Solh AA. Screening for obstructive sleep apnea in veterans with ischemic heart disease using a computerbased clinical decision-support system. Clin Res Cardiol 2012;101: 737-44.

22 Suhasini A, Palanivel P, Ramalingam V. Multimodel decision support system for psychiatry problems. Expert Syst Appl 2011;38:4990-7.

23 Kawamoto K, Del Fiol G, Lobach DF, Jenders RA. Standards for scalable clinical decision support: need, current and emerging standards, gaps, and proposal for progress. Open Med Inform J 2010;4:235-44.

24 Health Level Seven International, 2014. www.hl7.org [Accessed 24 June 2014].

25 SNOMED Clinical Terms ${ }^{\circledR}$ (SNOMED CT $\left.{ }^{\circledR}\right)$, 2014. www.nlm.nih. gov/research/umls/Snomed/snomed_main.html [Accessed 28 February 2014].

26 Wright A, Sittig DF, Ash JS et al. Clinical decision support capabilities of commercially-available clinical information systems. J Am Med Inform Assoc 2009;16:637-44.

27 Shibl R, Lawley M, Debuse J. Factors influencing decision support system acceptance. Decis Support Syst 2013;54:953-61.

28 Younger P. Internet-based information-seeking behaviour amongst doctors and nurses: a short review of the literature. Health Info Libr J 2010;7:2-10.

29 Kawamoto K, Lobach DF, Willard HF, Ginsburg GS. A national clinical decision support infrastructure to enable the widespread and consistent practice of genomic and personalized medicine. BMC Med Inform Decis Mak 2009;9:17.

30 Rai A, Chen L, Pye J, Baird A. Understanding determinants of consumer mobile health usage intentions, assimilation, and channel preferences. J Med Internet Res 2013;15:e149.

31 McCracken M, Godin S, Dalandan S. Quality assurance assessment of downloadable applications in health promotion and preventive healthcare. Poster presented at the 141 st annual meeting and exposition of the American Public Health Association, 2-6 November 2013, Boston, MA, USA (Poster 282584).

Address for correspondence: Dr D O'Sullivan, Centre for Health Informatics, School of Informatics, City University London, Northampton Square, London EC1V OHB.

Email: Dympna.0’Sullivan.1@city.ac.uk. 